\title{
Prognostic value of mean platelet volume to plateletcrit ratio in patients with osteosarcoma
}

This article was published in the following Dove Medical Press journal: Cancer Management and Research

\author{
Bo Gou \\ Hong Cao \\ Xinghua Cheng \\ Wei Shang \\ Mingqing $\mathrm{Xu}$ \\ Wei Qian
}

Department of Orthopedics, Renmin Hospital, Hubei University of

Medicine, Shiyan 442000, Hubei, China

Correspondence: Wei Qian

Department of Orthopedics, Renmin Hospital, Hubei University of Medicine, Chaoyang Road 39th, Shiyan 442000,

Hubei, China

$\mathrm{Tel} / \mathrm{Fax}+867198637323$

Email weiqiansy@।63.com
Objective: The objective of this study was to investigate the prognostic value of preoperative mean platelet volume to plateletcrit (MPV/PCT) ratio in patients with osteosarcoma.

Materials and methods: The retrospective study included 188 consecutive osteosarcoma patients who experienced neoadjuvant chemotherapy and surgical resection of tumor. The relationships between MPV/PCT and clinicopathological characteristics were analyzed. The Kaplan-Meier analysis and Cox regression proportional hazard model were applied to assess the prognostic value of MPV/PCT ratio.

Results: MPV/PCT ratio was found to be significantly associated with platelet count, platelet distribution width, and PCT (all $P<0.001$ ). Kaplan-Meier analysis showed that high MPV/ $\mathrm{PCT}$ ratio $(\geq 43.58)$ was associated with a prolonged disease-free survival (DFS, $P=0.035)$. The multivariate Cox revealed that only good chemotherapy response was an independent predictor of better DFS in the overall population. However, the stratification analysis showed that a high MPV/PCT ratio $(\geq 43.58)$ was indicated as an independent prognostic factor for a favorable DFS (HR $=0.137,95 \% \mathrm{CI}=0.029-0.644, P=0.012)$ in the male osteosarcoma patients but not in female patients.

Conclusion: The high preoperative MPV/PCT ratio may serve as an independent prognostic factor for a favorable prognosis in male osteosarcoma patients. Further studies are needed to confirm the findings.

Keywords: osteosarcoma, MPV/PCT, prognosis, platelet indices

\section{Introduction}

Osteosarcoma represents the most common malignancy of bone, predominantly affecting adolescents and young adults. ${ }^{1}$ Despite substantial progress including the introduction of multidisciplinary treatment achieved in the diagnosis and treatment of osteosarcoma in the past decades, the 5-year overall survival (OS) in patients without metastasis approaches $50 \%-70 \%$ superior to $10 \%$ with the surgery treatment available earlier. ${ }^{2}$ Although traditional prognostic indicators such as Enneking surgical criteria, TNM staging, and alkaline phosphatase have been long-tested, heterogeneous clinical outcomes are frequently presented in clinical practice, ${ }^{3}$ suggesting the lack of accuracy and adequacy.

Recently, more attention has been focused on the clinical significance of platelet indices in various malignancies due to the inflammation bridging platelet and cancer. The growing evidence revealed that activated platelet could secret inflammatory factors such as chemokines, cytokines, and coagulation factors, which may intensify the 
recruitment of leukocytes such as monocytes, neutrophils, and eosinophils. ${ }^{4}$ Meanwhile, enhanced inflammation has been shown to facilitate cancer deterioration by sustaining proliferative signals, resisting cell death, inducing angiogenesis, activating invasion and metastasis, evading immune detection, and supporting cancer stem cells. ${ }^{5}$ Generally, platelet activity can be assessed by several platelet indices including mean platelet volume (MPV), platelet distribution width (PDW), platelet count (PLT), plateletcrit (PCT), and platelet large cell ratio (P-LCR), ${ }^{6,7}$ which are easily measurable parameters in complete blood count in clinical practice. In this regard, the prognostic role of these platelet indices have been reported in various cancers. Decreased MPV has been demonstrated to predict a poor prognosis in rectal cancer ${ }^{8}$ multiple myeloma, ${ }^{9}$ renal cell carcinoma, ${ }^{10}$ invasive bladder cancer, ${ }^{11}$ esophageal cancer, ${ }^{12}$ and diffuse large B-cell lymphoma, ${ }^{13}$ while increased PCT has been found to be associated with unfavorable prognostic factors in pancreatic adenocarcinoma, ${ }^{14}$ colorectal cancer, and endometrial cancer. ${ }^{15,16}$ Moreover, the prognostic value of combined platelet indices such as platelet distribution width-to-platelet count ratio (PDW/PLT), combination of the preoperative PLT and MPV (COP-MPV), has also been shown in various types of cancer by published studies. ${ }^{17-19}$ Although the previous study failed to present the prognostic role of PDW and PLT in osteosarcoma patients, the influence of MPV, PCT, and MPV to PCT ratio (MPV/PCT) on the prognosis of osteosarcoma patient has not been determined. Hence, the current study aimed to evaluate the prognostic significance of MPV/PCT in patients with osteosarcoma.

\section{Materials and methods Study design and population}

The study population was derived from a public dataset provided by Li et al, ${ }^{20}$ and the research ethic was no longer needed due to the public policy statement of the dataset which has also obtained the approval of research ethic in the original study. In this retrospective study, all patients with osteosarcoma were histologically confirmed following neoadjuvant chemotherapy and surgical resection. A Microsoft Excel Sheet was designed to collect the following clinicopathological information: age at diagnosis, gender, size and site of tumor, TNM and American Joint Committee on Cancer (AJCC) stage, and tumor necrosis rate in surgery samples following neoadjuvant chemotherapy. The laboratory data on preoperative MPV, PCT, PDW, and PLT were also collected. The primary outcome information was disease-free survival (DFS). The follow-up period varied from 1 to 63 months, with a median of 33 months. With a retrospective nature, the treatment intervention for each patient was not included for the analysis due to the lack of sufficient complete information record.

\section{Definition and optimal cutoff values for the studied platelet indices}

MPV/PCT was defined as the MPV value (fL) divided by PCT value (\%). The purpose of the study was to explore the prognostic value of MPV, PCT, PDW and PLT; the optimal cutoff values with prognostic significance were suggested by the cutoff finder which is a comprehensive and straightforward web application enabling rapid biomarker cutoff optimization. ${ }^{21}$ In case of no available significant discriminated value, the optimal cutoff point was set as the upper limit of normal for continuous variables. Accordingly, in this study, the optimal cutoff values for MPV, PCT, and PLT were set as that suggested by the cutoff finder, while the optimal cutoff value for PDW was set as the upper limit of normal.

\section{Statistical analyses}

All statistical analyses were performed by SPSS 17.0 statistical software. The continuous measurement data were described as mean $\pm \mathrm{SD}$ or median (range) based on the presence or absence of normal distribution, and the variables were categorized according to the optimal cutoff value. To compare with the baseline characteristics, the MPV/PCT ratio was divided into two groups by the suggested optimal cutoff value (43.58) of the cohort: high $(\geq 43.58)$ and low $(<43.58)$ levels. The associations between MPV/PCT and the clinical parameters were evaluated by the chi-squared test. DFS was defined as the interval from the date of surgery to the date of disease recurrence or progression or last follow-up. The survival curves were plotted by the Kaplan-Meier method, and the significance was assessed by the log-rank test. The univariate and multivariate Cox regression proportional hazard model analyses were further performed to evaluate the prognostic value of MPV/PCT in osteosarcoma patients. Stratification analysis was performed to explore the possible confounding effect on the association between MPV/PCT and the survival by adjusting the parameters found to be significant in the univariate analysis. All the statistical analyses were considered statistically significant with a two-tailed $P$-value $<0.05$.

\section{Results}

\section{Patient characteristics and analysis of preoperative MPV/PCT ratio}

A total of 188 osteosarcoma patients were included in the current study. The detailed characteristics of participants 
are shown in Table 1. There were 5.3\% (10/188), 93.6\% (176/188), and $1.1 \%(2 / 188)$ patients with stage I, II, and III cancer, respectively, and 58.5\% (110/188), 34.6\% (65/188), and $6.95 \%$ (13/188) of tumor originated from the femur, tibia, and fibula site, respectively. According to the established criterion, the optimal cutoff values for PLT, PDW, PCT, and MPV were set as 223 (high: $>223$, low: $\leq 223$ ), 16.3 (high: $\geq 16.3$, low: $<16.3$ ), 0.2635 (high: $>0.2635$, low: $\leq 0.2635$ ), and 10.25 (high: $\geq 10.25$, low: $<10.25$ ), respectively. The preoperative median of MPV/PCT ratio in the study was 43.73 with a range from 20.45 to 287.5 . The continuous variables were categorized as high and low group according to the optimal cutoff value. As shown in Table 1, a significant association of MPV/PCT ratio with PLT $(P<0.001)$, PDW $(P<0.001)$, and PCT $(P<0.001)$ was observed. Moreover, a borderline significant association between MPV/PCT ratio and patient age at the diagnosis was also found $(P=0.051)$. The associations between MPV/PCT ratio and clinical parameters are summarized in Table 1.

\section{DFS and preoperative MPV/PCT ratio}

With a median follow-up duration of 33 months, 78 (41.5, $78 / 188$ ) patients experienced recurrence or progression. As shown in Table 2, the univariate Cox regression analysis revealed a significant association between chemotherapy response $(\mathrm{HR}=0.382,95 \% \mathrm{CI}=0.196-0.743, P=0.005)$, PLT $(\mathrm{HR}=1.665,95 \% \mathrm{CI}=1.044-2.657, P=0.032)$, PCT $(\mathrm{HR}$ $=1.669,95 \% \mathrm{CI}=1.069-2.607, P=0.024)$, and MPV $/ \mathrm{PCT}$ (HR $=0.620,95 \% \mathrm{CI}=0.394-0.977, P=0.039)$ and DFS in the overall population. To identify the independent prognostic factors, significant factors revealed by the univariate analysis were included in the multivariate analysis. The results of the

Table I Relationships between clinical and laboratory data and MPV/PCT in patients with osteosarcoma

\begin{tabular}{|c|c|c|c|c|}
\hline \multirow[t]{2}{*}{ Variables } & \multirow[t]{2}{*}{ Total } & \multicolumn{2}{|c|}{ MPV/PCT $\left(\times 10^{2} \mathrm{fL}, \mathrm{N}=188\right)$} & \multirow[t]{2}{*}{$P$-value } \\
\hline & & $\geq 43.58$ & $<43.58$ & \\
\hline Age $(>18 / \leq 18$, years $)$ & - & $46 / 49$ & $32 / 61$ & 0.051 \\
\hline Gender (male/female) & - & $68 / 27$ & $58 / 35$ & 0.179 \\
\hline Site (femur/tibia/fibula) & - & $52 / 35 / 8$ & $58 / 30 / 5$ & 0.501 \\
\hline Size $(\geq 7 /<7 \mathrm{~cm})$ & - & $43 / 52$ & $48 / 45$ & 0.384 \\
\hline $\mathrm{T}$ stage $(\mathrm{TI} / \mathrm{T} 2)$ & - & $87 / 8$ & $85 / 8$ & 0.965 \\
\hline Response (good/poor) & - & $21 / 74$ & $22 / 7 \mid$ & 0.800 \\
\hline AJCC stage (IIB-III/I-IIA) & - & $38 / 57$ & $46 / 47$ & 0.192 \\
\hline PLT $\left(>223 / \leq 223 \times 10^{9} / L\right)$ & $238 \pm 69.7$ & $8 / 87$ & $93 / 0$ & $<0.001$ \\
\hline $\operatorname{PDW}(\geq 16.3 /<16.3 \mathrm{fL})$ & $13.2 \pm 2.66$ & $28 / 67$ & $5 / 88$ & $<0.001$ \\
\hline PCT $(>0.2635 \% / \leq 0.2635 \%)$ & $0.246 \pm 0.074$ & $9 / 86$ & $61 / 32$ & $<0.001$ \\
\hline $\operatorname{MPV}(\geq 10.25 /<10.25 \mathrm{fL})$ & $10.42 \pm 1.86$ & $54 / 41$ & $47 / 46$ & 0.386 \\
\hline
\end{tabular}

Note: The bold data indicate the statistical significance of relationships.

Abbreviations: AJCC, American Joint Committee on Cancer; MPV, mean platelet volume; PCT, plateletcrit; PDW, platelet distribution width; PLT, platelet count.

Table 2 Univariate and multivariate Cox regression analysis for DFS

\begin{tabular}{|c|c|c|c|c|}
\hline \multirow[t]{2}{*}{ Variables } & \multicolumn{2}{|c|}{ Univariate analysis for DFS } & \multicolumn{2}{|c|}{ Multivariate analysis for DFS } \\
\hline & HR (95\%Cl) & $P$-value & HR (95\%Cl) & $P$-value \\
\hline Age ( $>18$ vs $\leq 18$ years $)$ & $0.830(0.524-1.315)$ & 0.427 & & \\
\hline Gender (male vs female) & $0.988(0.619-1.575)$ & 0.959 & & \\
\hline Site (femur vs non-femur) & $1.240(0.780-1.971)$ & 0.362 & & \\
\hline Size $(\geq 7 /<7 \mathrm{~cm})$ & $1.227(0.786-1.915)$ & 0.368 & & \\
\hline T stage (TI vs T2) & $1.583(0.639-3.917)$ & 0.321 & & \\
\hline Response (good vs poor) & $0.382(0.196-0.743)$ & 0.005 & $0.355(0.180-0.700)$ & 0.003 \\
\hline AJCC stage (IIB-III vs I-IIA) & $1.289(0.824-2.019)$ & 0.266 & & \\
\hline PLT (>223 vs $\left.\leq 223 \times 10^{9} / L\right)$ & $1.665(1.044-2.657)$ & 0.032 & $1.895(0.653-5.50 \mathrm{I})$ & 0.240 \\
\hline PDW $(\geq 16.3$ vs $<16.3 \mathrm{fL})$ & $1.505(0.878-2.578)$ & 0.137 & & \\
\hline PCT $(>0.2635 \%$ vs $\leq 0.2635 \%)$ & $1.669(1.069-2.607)$ & 0.024 & $1.537(0.895-2.639)$ & 0.120 \\
\hline $\operatorname{MPV}(\geq 10.25$ vs $<10.25 \mathrm{fL})$ & $0.879(0.563-1.372)$ & 0.570 & & \\
\hline MPV/PCT $\left(\geq 43.58\right.$ vs $\left.<43.58 \times 10^{2} \mathrm{fL}\right)$ & $0.620(0.394-0.977)$ & 0.039 & $0.697(0.237-2.052)$ & 0.512 \\
\hline
\end{tabular}

Note: The bold data indicate the statistical significance of relationships.

Abbreviations: AJCC, American Joint Committee on Cancer; DFS, disease-free survival; MPV, mean platelet volume; PCT, plateletcrit; PDW, platelet distribution width; PLT, platelet count. 
multivariate analyses qualified that only a good chemotherapy response was independently associated with a better DFS (HR $=0.355,95 \% \mathrm{CI}=0.180-0.700, P=0.003)$. To further explore the effect of confounding factors on the prognostic role of MPV/PCT, the stratification analysis was performed by adjusting the parameters which were found to be significant in the

Table 3 Stratification analysis concerning the prognostic value of MPV/PCT ratio by multivariate Cox regression analysis

\begin{tabular}{|c|c|c|}
\hline $\begin{array}{l}\text { MPV/PCT ratio } \\
\text { (high vs low) }\end{array}$ & $\operatorname{HR}(95 \% \mathrm{Cl})^{a}$ & $P$-value \\
\hline \multicolumn{3}{|l|}{ Age } \\
\hline$>18$ years $(\mathrm{N}=78)$ & $0.988(0.206-4.750)$ & 0.988 \\
\hline$\leq 18$ years $(N=1 \mid 0)$ & $0.303(0.064-1.429)$ & 0.131 \\
\hline \multicolumn{3}{|l|}{ Gender } \\
\hline Male $(\mathrm{N}=126)$ & $0.137(0.029-0.644)$ & 0.012 \\
\hline Female $(\mathrm{N}=62)$ & $0.583(0.126-2.695)$ & 0.490 \\
\hline \multicolumn{3}{|l|}{ Site } \\
\hline Femur $(\mathrm{N}=|\mathrm{I}| 0)$ & $0.690(0.192-2.475)$ & 0.569 \\
\hline Non-femur $(\mathrm{N}=78)$ & $0.638(0.078-5.22 I)$ & 0.675 \\
\hline \multicolumn{3}{|l|}{ Size } \\
\hline$\geq 7 \mathrm{~cm}(\mathrm{~N}=9 \mathrm{l})$ & $0.473(0.058-3.844)$ & 0.484 \\
\hline$<7 \mathrm{~cm}(\mathrm{~N}=97)$ & $0.415(0.110-1.561)$ & 0.193 \\
\hline \multicolumn{3}{|l|}{ Response } \\
\hline Poor $(\mathrm{N}=145)$ & $0.559(0.160-1.946)$ & 0.360 \\
\hline Good $(\mathrm{N}=43)$ & $0.643(0.078-5.330)$ & 0.683 \\
\hline \multicolumn{3}{|l|}{ AJCC stage } \\
\hline I-IIA (N=104) & $0.793(0.215-2.924)$ & 0.727 \\
\hline IIB-III ( $\mathrm{N}=84)$ & $0.778(0.085-7.108)$ & 0.824 \\
\hline
\end{tabular}

Notes: ${ }^{\text {HHR }}$ adjusted by response, PLT, and PCT which were significant in the overall population. The bold data indicate the statistical significance of relationships. Abbreviations: AJCC, American Joint Committee on Cancer; MPV, mean platelet volume; PCT, plateletcrit; PLT, platelet count. univariate analysis. As shown in Table 3, higher MPV/PCT ratio was found to be independently associated with a better DFS in male patients $(\mathrm{HR}=0.137,95 \% \mathrm{CI}=0.029-0.644$, $P=0.012$ ) but not in female patients. The Kaplan-Meier cumulative survival curves for DFS based on the MPV/PCT ratio and chemotherapy response are shown in Figure 1.

\section{Discussion}

In this study, we evaluated the prognostic role of MPV/PCT ratio in osteosarcoma patients. The finding of our study implied for the first time that higher MPV/PCT ratio might be associated with a good prognosis in male osteosarcoma.

It is well known that MPV is one of the most commonly used platelet indices for evaluating the platelet activity and function. ${ }^{22}$ While platelet activation plays an inducible role in the inflammatory process in cancerous diseases due to secreted pro-inflammatory factors, chemokines, and growth factors such as transforming growth factor- $\beta$, interleukin- $1 \beta$, monocyte chemoattractant protein-1, tumor necrosis factor- $\alpha$, vascular endothelial growth factor, platelet-derived growth factor, and fibroblast growth factor, which could promote tumor cell growth, angiogenesis, and metastasis. ${ }^{8,12,23,24}$ Numerous studies have also reported the association of MPV with prognosis in various types of cancer. Considering the strong linkage between inflammation and cancer, platelet-involved inflammation might be of major importance in understanding the association between MPV and survival in cancers. MPV is an early parameter of activated platelets. Large platelets are more reactive than their smaller
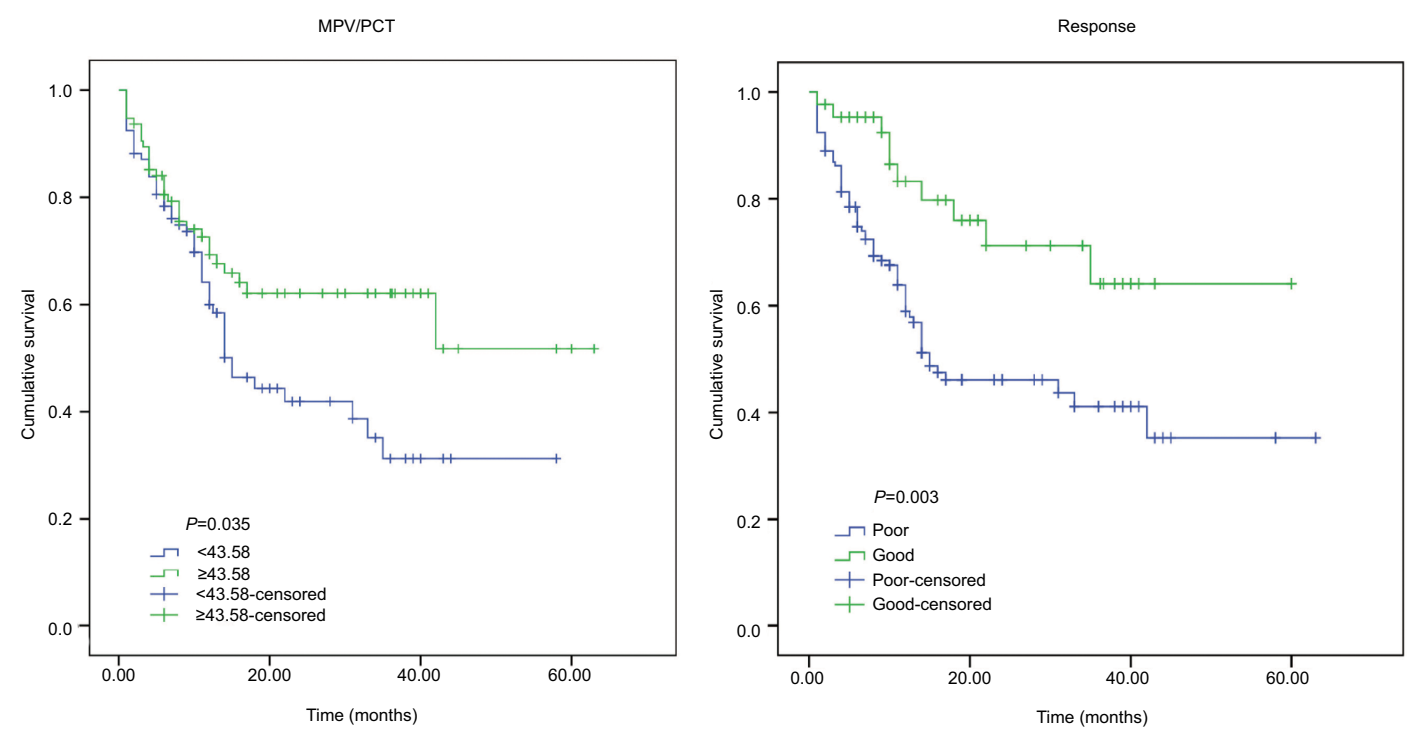

Figure I Kaplan-Meier curves for DFS by MPV/PCT ratio and chemotherapy response. Abbreviations: DFS, disease-free survival; MPV, mean platelet volume; PCT, plateletcrit. 
counterparts in releasing a variety of pro-inflammatory cytokines, are more likely to aggregate with more granules and receptors, and have more adhesive ability. Whereas, the release rate of small size platelets from the bone marrow was compensatorily increased since excessive pro-inflammatory cytokines may interfere with megakaryopoiesis, and this interfering may result in decreased production of large platelets. Therefore, lower MPV level may suggest an enhanced consumption of large platelets and increased inflammatory activity. In support of this, recent studies demonstrated an association of low levels of MPV with high-grade inflammatory diseases and an elevated MPV in the course of antiinflammatory therapy. Moreover, most studies have also revealed a negative effect of low MPV on the survival in rectal cancer, ${ }^{8}$ multiple myeloma, ${ }^{9}$ renal cell carcinoma, ${ }^{10}$ invasive bladder cancer, ${ }^{11}$ esophageal cancer ${ }^{12}$ and diffuse large B-cell lymphoma. ${ }^{13}$ In addition, PCT is also an important platelet index, reflecting the total platelet mass. ${ }^{25}$ It is not a frequently used biomarker, but we believed that the importance of PCT has been underestimated. Although knowledge about PCT is limited in the literature, previous studies have also demonstrated an association of PCT with certain inflammatory diseases and vascular diseases ${ }^{26,27}$ and an adverse impact on the clinical outcomes in patients with myocardial infarction. ${ }^{25,28}$ PCT level was also found higher in endometrial cancer, ${ }^{16}$ papillary thyroid carcinoma, ${ }^{29}$ lung cancer, ${ }^{30}$ pancreatic adenocarcinoma, ${ }^{14}$ and colorectal cancer ${ }^{15}$ and was associated with a shorter survival in pancreatic adenocarcinoma. ${ }^{14}$ In this study, we found an inverse association of MPV/PCT with PLT and PDW which were both reported to be associated with poor prognosis in most cancers, and we also presented an association of elevated MPV/PCT with better prognosis in osteosarcoma in the univariate analysis, suggesting the potential prognostic value although it was not revealed as an independent predictor by the multivariate Cox analysis in the overall population. Considering the negative and positive effects of a low MPV and PCT, respectively, on the survival of most cancers, our finding that supported a survival benefit for MPV/PCT was plausible. In keeping with our finding, MPV/PCT has previously been found to be a predictor of in-hospital pediatric mortality. ${ }^{31}$ Nonetheless, the mechanism underlying the association of MPV/PCT ratio with prognosis in osteosarcoma patients remains unclear.

Despite the absence of a significant independent prognostic role of MPV/PCT ratio in the overall population, the stratification analysis showed that the MPV/PCT ratio may be independently associated with DFS in male osteosarcoma patients, suggesting the potential importance of sex difference on the concerned association. One possible explanation could be that platelet activity and function may differ between male and female. Studies have found that women had higher platelet counts than men. ${ }^{32,33}$ The platelet aggregation induced by collagen was stronger in male rats than in females, and the activity of platelet cyclooxygenases which is a common inflammatory mediator was higher in males than in females. ${ }^{34}$ Moreover, a previous study has also found that males are more susceptible to arachidonate-induced platelet diminution than females, which is related to a sex difference in mouse platelet function. ${ }^{35} \mathrm{~A}$ clinical study reported that aspirin may be less effective at inhibiting platelet aggregation in women compared with men who have a history of ischemic stroke or TIA. ${ }^{36}$ The platelet parameter could be affected by estrogen in female population. Previous studies always focused on the typical pathological alteration of platelet parameter, neglecting the sex-specific difference. In female adults, estrogen, which functions as anti-inflammatory agent, may underlie the potential biochemical mechanism. ${ }^{37}$ Reportedly, autocrine estrogen was able to trigger the platelet production, and the inhibition of platelet production by estrogen receptor antagonists in vivo also supported such a role. ${ }^{38}$ Nevertheless, the effect of estrogen on the MPV/PCT ratio and on the association of MPV/PCT ratio with the clinical outcome of female osteosarcoma patients remain for further investigation. Collectively, the fluctuation of platelet parameter is potentially neutralized by the potentiation of platelet production triggered by estrogen and attenuated by higher baseline in female at inflammatory state, while may be enhanced and easily affected due to the increased susceptibility to inducers such as inflammation in male, which may contribute to the discrepancy for the association concerned.

Several limitations should be noticed in the present study. First, the association of MPV/PCT ratio with OS was not determined due to the lack of sufficient data. Second, the treatment regimen and therapy strategy varied between individuals, which may lead to difference in therapeutic response, and thus accounted for discrepant prognosis. However, the treatment intervention was not included for the analysis due to the lack of sufficient complete information, which was likely a considerable limitation for this study. Third, the small sample size and single-center design may cause bias in terms of data selection and analysis, which may limit the reliability and robustness of results particularly in the subgroup. Fourth, the ethnicity difference for platelet parameter has been reported. ${ }^{32}$ However, the current study was based on data from Chinese, the application of the findings to other ethnic groups required further investigation. 


\section{Conclusion}

The present study suggests that preoperative MPV/PCT ratio may serve as an independent prognostic marker for male patients with osteosarcoma. In light of the limitations mentioned before, further studies are needed to confirm these findings.

\section{Acknowledgment}

All the authors declare that no funding was received regarding this work.

\section{Disclosure}

The authors report no conflicts of interest in this work.

\section{References}

1. Liu B, Huang Y, Sun Y, et al. Prognostic value of inflammation-based scores in patients with osteosarcoma. Sci Rep. 2016;6(1):39862.

2. Li YJ, Yao K, Lu MX, Zhang WB, Xiao C, Tu CQ. Prognostic value of the $\mathrm{C}$-reactive protein to albumin ratio: a novel inflammationbased prognostic indicator in osteosarcoma. Onco Targets Ther. 2017;10:5255-5261.

3. Xia WK, Liu ZL, Shen D, Lin QF, Su J, Mao WD. Prognostic performance of pre-treatment NLR and PLR in patients suffering from osteosarcoma. World J Surg Oncol. 2016;14(1):127.

4. Pitchford SC, Page CP. Platelet activation in asthma: integral to the inflammatory response. Clin Exp Allergy. 2006;36(4):399-401.

5. Franco AT, Corken A, Ware J. Platelets at the interface of thrombosis, inflammation, and cancer. Blood. 2015;126(5):582-588.

6. Song X, Zhu H, Pei Q, et al. Significance of inflammation-based indices in the prognosis of patients with non-metastatic colorectal cancer. Oncotarget. 2017;8(28):45178-45189.

7. Topal E, Celiksoy MH, Catal F, Karakoç HT, Karadağ A, Sancak R. The platelet parameters as inflammatory markers in preschool children with atopic eczema. Clin Lab. 2015;61(5-6):493-496.

8. Włodarczyk M, Kasprzyk J, Sobolewska-Włodarczyk A, et al. Mean platelet volume as a possible biomarker of tumor progression in rectal cancer. Cancer Biomark. 2016;17(4):411-417.

9. Zhuang Q, Xiang L, Xu H, et al. The independent association of mean platelet volume with overall survival in multiple myeloma. Oncotarget. 2016;7(38):62640-62646.

10. Yun ZY, Zhang X, Liu YS, et al. Lower mean platelet volume predicts poor prognosis in renal cell carcinoma. Sci Rep. 2017;7(1):6700.

11. Wang X, Cui MM, Xu Y, et al. Decreased mean platelet volume predicts poor prognosis in invasive bladder cancer. Oncotarget. 2017;8(40):68115-68122.

12. Shen W, Cui MM, Wang X, Wang R-T. Reduced mean platelet volume is associated with poor prognosis in esophageal cancer. Cancer Biomark. 2018;22(3):559-563.

13. Zhou S, Ma Y, Shi Y, et al. Mean platelet volume predicts prognosis in patients with diffuse large B-cell lymphoma. Hematol Oncol. 2018;36(1):104-109.

14. Wang L, Sheng L, Liu P. The independent association of platelet parameters with overall survival in pancreatic adenocarcinoma receiving intensity-modulated radiation therapy. Int J Clin Exp Med. 2015;8(11):21215-21221.

15. Zhu X, Cao Y, Lu P, et al. Evaluation of platelet indices as diagnostic biomarkers for colorectal cancer. Sci Rep. 2018;8(1):11814.

16. Karateke A, Kaplanoglu M, Baloglu A. Relations of platelet indices with endometrial hyperplasia and endometrial cancer. Asian Pac J Cancer Prev. 2015;16(12):4905-4908.
17. Zhang F, Chen Z, Wang P, et al. Combination of platelet count and mean platelet volume (COP-MPV) predicts postoperative prognosis in both resectable early and advanced stage esophageal squamous cell cancer patients. Tumor Biol. 2016;37(7):9323-9331.

18. Park JW, Kim CH, Ha YC, Kim MY, Park SM. Count of platelet and mean platelet volume score: serologic prognostic factor in patients with oral squamous cell carcinoma. J Korean Assoc Oral Maxillofac Surg. 2017;43(5):305-311.

19. Takeuchi H, Abe M, Takumi Y, et al. The prognostic impact of the platelet distribution width-to-platelet count ratio in patients with breast cancer. PLoS One. 2017;12(12):e0189166.

20. Li H, Wang Y, Liu Z. et al. Lack of association between platelet indices and disease stage in osteosarcoma at diagnosis. PLoS One. 2017;12(4):e174668.

21. Budczies J, Klauschen F, Sinn BV, et al. Cutoff finder: a comprehensive and straightforward web application enabling rapid biomarker cutoff optimization. PLoS One. 2012;7(12):e51862.

22. Gasparyan AY, Ayvazyan L, Mikhailidis DP, Kitas GD. Mean platelet volume: a link between thrombosis and inflammation? Curr Pharm Des. 2011;17(1):47-58.

23. Banks RE, Forbes MA, Kinsey SE, et al. Release of the angiogenic cytokine vascular endothelial growth factor (VEGF) from platelets: significance for VEGF measurements and cancer biology. Br J Cancer. 1998;77(6):956-964.

24. Senzel L, Gnatenko DV, Bahou WF. The platelet proteome. Curr Opin Hematol. 2009;16(5):329-333.

25. Gul M, Uyarel H, Akgul O, et al. Long-term prognostic significance of admission plateletcrit values in patients with non-ST elevation myocardial infarction. Blood Coagul Fibrinolysis. 2016;27(6):696-701.

26. Tang J, Gao X, Zhi M, et al. Plateletcrit: a sensitive biomarker for evaluating disease activity in Crohn's disease with low hs-CRP. $J$ Dig Dis. 2015;16(3):118-124.

27. Akpinar I, Sayin MR, Gursoy YC, et al. Plateletcrit and red cell distribution width are independent predictors of the slow coronary flow phenomenon. J Cardiol. 2014;63(2):112-118.

28. Uğur M, Ayhan E, Bozbay M, et al. The independent associatıon of plateletcrit with long-term outcomes in patients undergoing primary percutaneous coronary intervention. J Crit Care. 2014;29(6): 978-981.

29. Dincel O, Bayraktar C. Evaluation of platelet indices as a useful marker in papillary thyroid carcinoma. Bratisl Lek Listy. 2017;118(3):153-155.

30. Şahin F, Aslan A. Relationship between inflammatory and biological markers and lung cancer. J Clin Med. 2018;7(7):160.

31. Golwala ZM, Shah H, Gupta N, Sreenivas V, Puliyel JM. Mean platelet volume (MPV), platelet distribution width (PDW), platelet count and Plateletcrit (PCT) as predictors of in-hospital paediatric mortality: a case-control study. Afr Health Sci. 2016;16(2):356-362.

32. Segal JB, Moliterno AR. Platelet counts differ by sex, ethnicity, and age in the United States. Ann Epidemiol. 2006;16(2):123-130.

33. Stevens RF, Alexander MK. A sex difference in the platelet count. $\mathrm{Br}$ J Haematol. 1977;37(2):295-300.

34. Morikawa M, Kojima T, Inoue M, Tsuboi M, et al. Sex difference in the effect of aspirin on rat platelet aggregation and arachidonic acid metabolism. Jpn.J.Pharmacol. 1985;37(4):317-323.

35. Torres Duarte AP, Ramwell P, Myers A. Sex differences in mouse platelet aggregation. Thromb Res. 1986;43(1):33-39.

36. Cavallari LH, Helgason CM, Brace LD, Viana MA, Nutescu EA. Sex difference in the antiplatelet effect of aspirin in patients with stroke. Ann Pharmacother. 2006;40(5):812-817.

37. Wang LR, Zhou YF, Zhou YJ, et al. Elevation of plateletcrit increasing the risk of non-alcoholic fatty liver disease development in female adults: a large population-based study. Clin Chim Acta. 2017;474: $28-33$.

38. Nagata Y, Yoshikawa J, Hashimoto A, et al. Proplatelet formation of megakaryocytes is triggered by autocrine-synthesized estradiol. Genes Dev. 2003;17(23):2864-2869. 
Cancer Management and Research

\section{Publish your work in this journal}

Cancer Management and Research is an international, peer-reviewed open access journal focusing on cancer research and the optimal use of preventative and integrated treatment interventions to achieve improved outcomes, enhanced survival and quality of life for the cancer patient.

The manuscript management system is completely online and includes

Submit your manuscript here: https://www.dovepress.com/cancer-management-and-research-journal

a very quick and fair peer-review system, which is all easy to use. Visit $\mathrm{http}: / / \mathrm{www}$.dovepress.com/testimonials.php to read real quotes from published authors. 